\title{
Enigma of aortic aneurysms continues to be enigmatic!
}

\author{
Om Prakash Yadava ${ }^{1} \mathbb{D}$
}

Published online: 15 January 2022

(c) Indian Association of Cardiovascular-Thoracic Surgeons 2022

Frontiers in management of aortic disorders continue to advance and yet their enigma perseveres unabated. No wonder then, within two years of a special issue on 'Aortic Surgeries', we are bringing out yet another dedicated issue on the same theme.

To begin with, the pathogenesis of thoracic aortic aneurysm (TAA), one of the commonest aortic pathologies, remains a waffling conundrum. Besides age-related degeneration of the aortic wall, enhanced by systemic hypertension, complex aortopathy associated with congenital bicuspid aortic valves and various genetic perturbations and mutations have been implicated in the genesis of aneurysms of the aorta. Typically in a normal aorta, elastin and smooth muscle cells (SMCs) containing media provide elasticity and the collagen-rich adventitia provides the tensile strength. Because of the circumferential alignment of the collagen fibres, there is increased tensile strength in that direction, compared to the axial strength in aorta [1]. Any deficiency in collagen fibres [2] or apoptosis of SMCs may lead to development of TAA. Death of SMCs vitiates the balance between matrix metalloproteinases (MMPs) and their tissue inhibitors (TIMPs) [3]. The former leads to breakdown of collagen and elastin, which is counter-balanced by the TIMPs. Even energy storage during systolic expansion of the aorta is dependent on cross-linked elastin fibres [4] and may have a bearing on aneurysm formation. For TAAs, where an obvious causation in a form of a genetic disorder or a congenital malformation (bicuspid aortic valve) is not found, Humphrey et al. suggest that there may be mechano-sensing errors with loss of extracellular matrix (ECM) regulation [5].

Advanced diagnostics and contemporary imaging modalities have provided some insights into the mechanistic basis of aneurysm formation, though a lot still remain hypothetical and await endorsement. In vivo studies using speckle

Om Prakash Yadava

op_yadava@yahoo.com

1 National Heart Institute, New Delhi, India tracking have been used to measure aortic deformation and TAA strains [6]. Computed tomography (CT) and magnetic resonance imaging (MRI) provide insight into the flow patterns and the aortic wall stiffness, and their role in causation of aneurysms. Solid mechanics and modelling too have been used to study the wall stress in aorta in order to predict the risk of dissection [7]. However, due to heterogeneity of various pathologies, computational fluid dynamics (CFD) is being increasingly used to study blood flow, wall deformation, and potential for aneurysm formation [8]. 'These CFD studies demonstrate that small geometrical variations may cause large haemodynamic changes, greatly influencing vessel wall stresses and progression of TAAs' [1]. As each of these methods has limitations, it is important to judiciously combine them for accurate characterisation of the aortic wall, its structural composition, functional behaviour, and its proclivity to degeneration and aneurysm formation [1].

Even therapeutics remain a speculative quandary and the surgical thresholds are, at best, tentative. We have been conventionally treating aortic aneurysms surgically using simplistic indices of aneurysm size and rate of growth. Both of these, in reference to the mode of treatment and the indication of treatment, need to be revisited. The current recommendation for surgery based on a size of $>5 \mathrm{~cm}$ or an expansion of $>0.5 \mathrm{~cm} /$ year does not always hold prognostically true, as nearly half of dissections of the ascending aorta take place in vessels below the surgical thresholds [9]. This has led to the dawning that just the size of the aneurysm and its rate of progression are not enough for decision-making. Thus, biomechanics of the aortic wall, and the aneurysm, are extremely important for decision-making and various computational methods like CFD can be used to biomechanically characterise the aorta. 'However, any singular approach typically focuses on only the material properties of the aortic wall, intra-aneurysmal haemodynamics, or invivo vessel dynamics, neglecting combinatorial factors that influence aneurysm development and progression' [1]. There is thus an acute felt 'need for comprehensive approaches that combine multiple characterisation methods to study the mechanisms contributing to focal weakening and rupture' 
[1]. A caveat - Whenever one considers the rate of expansion of an aneurysm, one must also factor in age-related dilatation of the aorta, to an extent of $0.15 \mathrm{~mm} / \mathrm{year}$, which is perfectly normal.

Despite spectacular advances in the field, to trump it all and swivel the field on its head, we need a paradigmatic volte-face in our approach to aneurysms of the aorta. We need to look for causative pathways for their development and devise ways and means, including genetic manipulation and pharmacotherapy, to abort these pathways and to prevent aneurysm formation, so that the patients are channelled from surgical to medical approaches. Targeting inflammation through the tryptophan metabolism or kynurenine pathway is one such novel target [9]. Therefore, it is imperative to understand the molecular basis, and genetic and other regulatory networks, which trigger aneurysm formation, their subsequent expansion, dissection, and rupture. Genes which encode for proteins that regulate and govern SMCs and their contractile function (mechano-transduction) are important therapeutic targets, the full potential of which is yet to unfold.

Aortic disorders are one arena, where the clinicians need to actively partner and collaborate with researchers in the field of basic sciences so as to unravel the enigma, which still surrounds aortic disorders and their consequent disastrous outcomes. A major contributory issue has been that human studies are few and far between and animal models have limited applicability to humans. It is here that patient-derived induced pluripotent stem cells can be used to develop human models of aortic disorders. This 'disease-in-a-dish' may provide opportunities for evolving mechanistic insights to identify molecular and cellular basis and for innovations in pharmacotherapy for these disorders [10]. This may help in developing precision medicine and personalised treatment of aortic disorders. Another fast-evolving field of nanotechnology provides for 'ultrasensitive monitoring of cardiovascular markers by detecting and identifying aneurysms' [11] and for targeted drug delivery. Nanocarriers such as liposomes and gold and iron oxide can be used to deliver nanoparticles to target sites.

Backpedalling, notwithstanding the above, should an aneurysm develop, we need to further sophisticate our surgical techniques, including minimally invasive and endovascular approaches-more of it later in the current issue.

Foreseeable times are sure exciting and to give you a flavour, we present to you this compendium, guest edited and curated by our very own Drs. V.V. Bashi and M. Idhrees and contributed by oracles in their respective arena.

Dig deep and savour!
Funding None.

\section{Declarations}

Informed consent Not required.

Conflict of interest Nil.

\section{References}

1. Cebull HL, Rayz VL, Goergen CJ. Recent advances in biomechanical characterisation of thoracic aortic aneurysms. Front. Cardiovasc Med. 2020. https://doi.org/10.3389/fcvm.2020.00075.

2. Thunes JR, Phillippi JA, Gleason TG, Vorp DA, Maiti S. Structural modeling reveals microstructure-strength relationship for human ascending thoracic aorta. J Biomech. 2018;71:84-93. https://doi.org/10.1016/j.jbiomech.2018.01.037.

3. Lesauskaite V, Tanganelli P, Sassi C, et al. Smooth muscle cells of the media in the dilatative pathology of ascending thoracic aorta: morphology, immunoreactivity for osteopontin, matrix metalloproteinases, and their inhibitors. Hum Pathol. 2001;32:1003-11. https://doi.org/10.1053/hupa.2001.27107.

4. Kim J, Staiculescu MC, Cocciolone AJ, Yanagisawa H, Mecham RP, Wagenseil JE. Crosslinked elastic fibers are necessary for low energy loss in the ascending aorta. J Biomech. 2017;61:199-207. https://doi.org/10.1016/j.jbiomech.2017.07.011.

5. Humphrey JD, Milewicz DM, Tellides G, Schwartz MA. Dysfunctional mechanosensing in aneurysms. Science. 2014;344:477-9. https://doi.org/10.1126/science.1253026.

6. Alreshidan M, Shahmansouri N, Chung J, et al. Obtaining the biomechanical behavior of ascending aortic aneurysm via the use of novel speckle tracking echocardiography. J Thorac Cardiovasc Surg. 2017;153:781-8. https://doi.org/10.1016/j.jtcvs.2016.11. 056.

7. Xuan Y, Wang Z, Liu R, et al. Wall stress on ascending thoracic aortic aneurysms with bicuspid compared with tricuspid aortic valve. J Thorac Cardiovasc Surg. 2018;156:492-500. https://doi. org/10.1016/j.jtcvs.2018.03.004.

8. Youssefi P, Gomez A, Arthurs C, Sharma R, Jahangiri M, Alberto Figueroa C. Impact of patient-specific inflow velocity profile on hemodynamics of the thoracic aorta. J Biomech Eng. 2018. https://doi.org/10.1115/1.4037857.

9. Ramprasath T, Han Y-M, Zhang D, Yu C-J, Zou M-H. Tryptophan catabolism and inflammation: A novel therapeutic target for aortic diseases. Front Immunol. 2021. https://doi.org/10.3389/fimmu. 2021.731701.

10. Davaapil H, Shetty DK, Sinha S. Aortic, "disease-in-a-dish": mechanistic insights and drug development using iPSC-based disease modeling. Front Cell Dev Biol. 2020. https://doi.org/10. 3389/fcell.2020.550504.

11. Zhu S, Zhu K, Li J, Lai H, Wang C. Nano-biomaterials for the delivery of therapeutic and monitoring cues for aortic diseases. Front Bioeng Biotechnol. 2020. https://doi.org/10.3389/fbioe. 2020.583879 .

Publisher's note Springer Nature remains neutral with regard to jurisdictional claims in published maps and institutional affiliations. 